\title{
The molecular changes driving the carcinogenesis in Barrett's esophagus: Which came first, the chicken or the egg?
}

\author{
A. Russo $^{\mathrm{a}, *, 1}$, G. Bronte $^{\mathrm{a}, 1}$, D. Cabibi ${ }^{\mathrm{b}}$, V. Bazan $^{\mathrm{a}}$, G. Cicero $^{\mathrm{a}}$, A. Bertani $^{\mathrm{c}}$, \\ S. Rizzo ${ }^{\text {a }}$, E. Fiorentino ${ }^{\text {d }}$

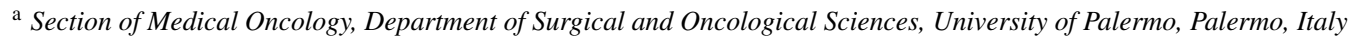 \\ ${ }^{\mathrm{b}}$ Department of Human Pathology, University of Palermo, Palermo, Italy \\ ${ }^{\mathrm{c}}$ Mediterranean Institute for Transplantation and Advanced Specialized Therapies (IsMeTT), Palermo, Italy \\ d Section of Oncological Surgery, Department of Surgical and Oncological Sciences, University of Palermo, Palermo, Italy
}

Accepted 10 December 2012

\section{Contents}

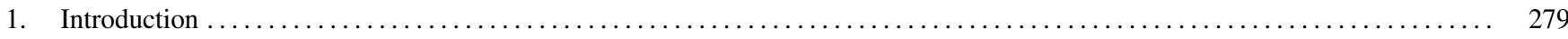

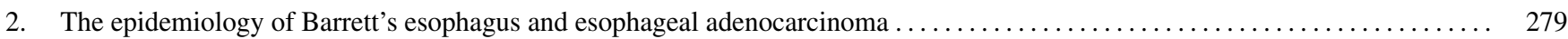

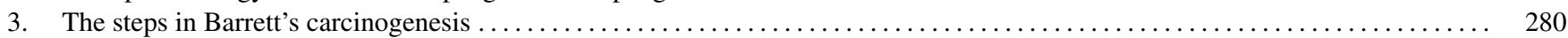

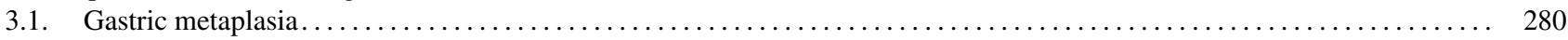

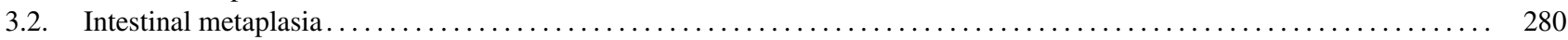

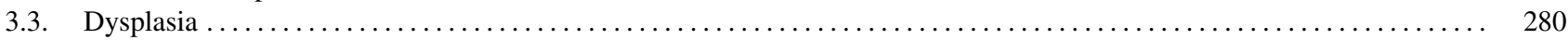

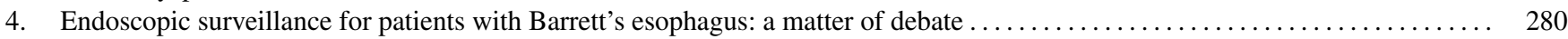

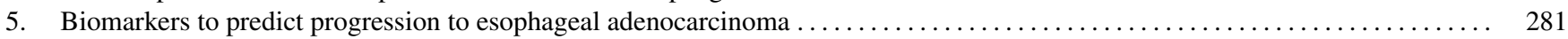

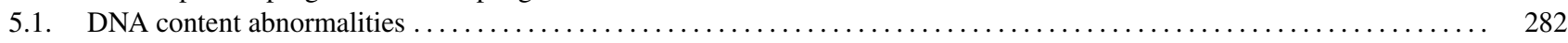

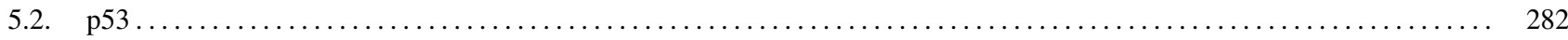

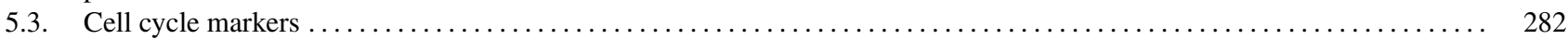

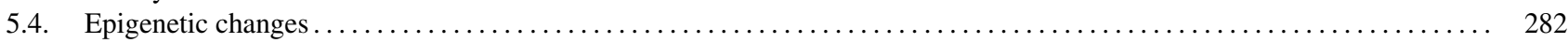

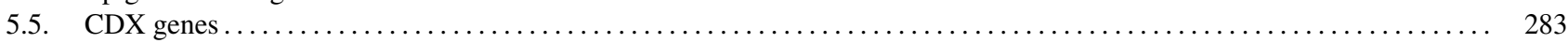

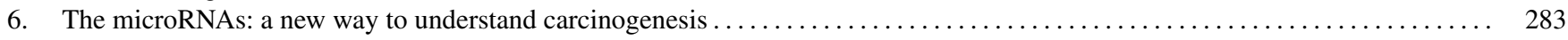

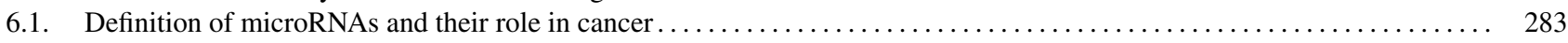

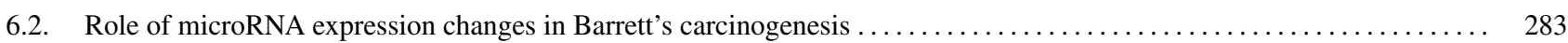

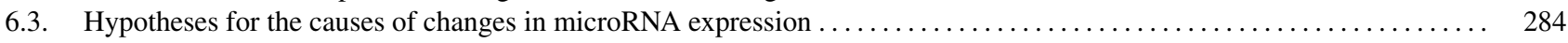

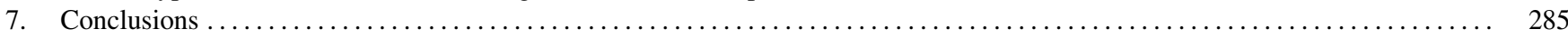

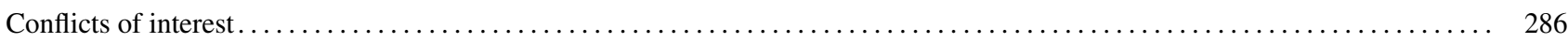

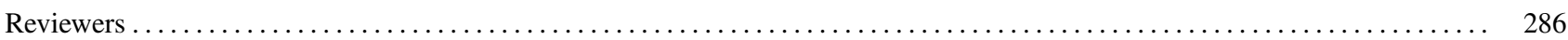

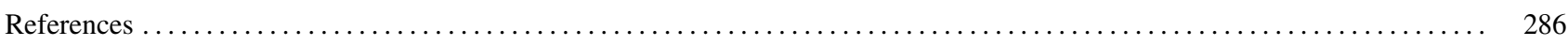

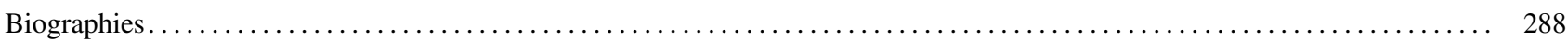

\begin{abstract}
Esophageal adenocarcinoma originates from columnar metaplastic epithelium of the distal esophagus. Various steps for this carcinogenetic process are known. Before the onset of high-grade dysplasia and adenocarcinoma, endoscopic surveillance is possible. However, because of the high cost of long-term surveillance, predictive factors for cancer are being evaluated to identify subjects with metaplasia who have a higher risk of developing malignancy. Molecular changes seem suitable for this purpose, but could require a high resource expenditure. While
\end{abstract}

\footnotetext{
* Corresponding author at: Section of Medical Oncology, Department of Surgical and Oncological Sciences, Università di Palermo, Via del Vespro 129, 90127 Palermo, Italy. Tel.: +39 0916552500; fax: +39 0916554529.

E-mail address: antonio.russo@usa.net (A. Russo).

1 These authors contributed equally to this work.
} 
trying to identify the best predictive factors for cancer risk, molecular changes and differences in miRNA expression profile between the various steps leading to cancer could help to clarify Barrett's carcinogenesis. In this attempt to find a molecular explanation for the onset of esophageal adenocarcinoma, it is still difficult to understand whether the molecular changes are causes or effects of the neoplastic phenotypic modifications.

(C) 2013 Elsevier Ireland Ltd. All rights reserved.

Keywords: Barrett's esophagus; Metaplasia; Dysplasia; Adenocarcinoma; Endoscopic surveillance; miRNAs

\section{Introduction}

Barrett's esophagus (BE) is characterized by the replacement of the normal squamous epithelium by a columnar-lined epithelium in the distal portion of the esophagus [1,2]. When the esophagus is chronically exposed to gastric reflux, a protective response is initiated which leads to metaplasia $[3,4]$.

BE can be diagnosed when a segment of columnar metaplasia of any length is found by endoscopic detection above the esophagogastric junction, but it needs to be confirmed by histological analysis [5]. This condition is most frequently associated with long-standing gastroesophageal reflux disease (GERD). Whilst this represents the main precursor lesion for the development of esophageal adenocarcinoma (EA), it can progress through various grades of dysplasia before the development of cancer [6]. However, despite this relationship between GERD, BE and EA, symptomatic GERD is infrequent or absent in $40-48 \%$ of people who develop EA.

The increasing prevalence of esophageal adenocarcinoma justifies the increased interest in its precursor lesion, BE. Several variations in gene expression show a relationship with its progression to cancer. However, the current sequence of molecular events is as yet unknown, and we lack an explanation for Barrett's carcinogenesis.

\section{The epidemiology of Barrett's esophagus and esophageal adenocarcinoma}

In Western countries GERD affects $10-20 \%$ of the population. The incidence is approximately 5 per 1000 persons per year [7]. BE is usually found in middle-aged adults. The prevalence of $\mathrm{BE}$ ranges from $0.9 \%$ to $4.5 \%$ of the general population. The prevalence of endoscopic BE (columnarlined epithelium) in reflux-disease patients referred for endoscopy usually ranges from $10 \%$ to $15 \%$ in Western countries, and $1.6 \%$ of them have histologically confirmed intestinal metaplasia [8,9].

The prevalence of EA has been increasing significantly in Western countries [10,11]. In 2005 there were 497,700 new cases, and the prevalence is expected to increase worldwide by approximately $140 \%$ by 2025 . EA has a poor prognosis, with the overall 5-year survival remaining less than $20 \%$; 416,500 people are estimated to have died from esophageal cancer in 2005 [12]. Whilst worldwide squamous-cell carcinoma is the most common histotype, in many Western countries adenocarcinoma has become the most prevalent form of esophageal cancer. This phenomenon represents the most dramatic epidemiological shift ever recorded, since esophageal adenocarcinoma has gone from being an unknown disease until the 1950s to the fastest increasing cancer in America in the 2000s [13]. New patients will be diagnosed with esophageal cancer, and more than $50 \%$ of cases will be adenocarcinoma. This increasing incidence involves all disease stages and all ages, but the greatest increase is in men over 65 years old.

A recent English publication concerning the incidence and survival of esophageal cancer reported that the incidence of this malignancy increased until 2002, then remained relatively stable, whereas gastric cancer declined over this period [14].

$\mathrm{BE}$ has been considered a strong risk factor for EA with an assumed risk of $0.5 \%$, but when only high-quality epidemiological studies are analyzed the EA incidence in BE is $0.39 \%$ and even lower, at least in Europe, with an absolute annual risk of $0.12 \%[15,16]$.

Obesity, defined as body mass index (BMI) $>30 \mathrm{~kg} / \mathrm{m}^{2}$, is also a clear risk factor for EA. Two recent metaanalyses have estimated relative risks for developing cancer of between 2.4 and 2.8 for those with a BMI $>30 \mathrm{~kg} / \mathrm{m}^{2}$ (obese) and between 1.5 and 1.8 for those considered overweight $\left(\mathrm{BMI}=25.0-29.9 \mathrm{~kg} / \mathrm{m}^{2}\right)[17,18]$.

Some preliminary findings suggest a correlation between GERD and obesity. For this reason obese people with symptomatic GERD had a substantially higher risk for EA (odds ratio $[\mathrm{OR}]=16.5,95 \% \mathrm{CI}=8.9-30.6)$ than people with obesity but no reflux $(\mathrm{OR}=2.2,95 \% \mathrm{CI}=1.1-4.3)$ or reflux but no obesity $(\mathrm{OR}=5.6,95 \% \mathrm{CI}=2.8-11.3)$ compared to people with a healthy BMI and no reflux symptoms [19].

Other weaker risk factors for EA include cigarette smoking, which approximately doubles the risk, and a diet low in fruits and vegetables. Alcohol does not appear to have an important role in EA. Infection with Helicobacter pylori is related to a reduced EA risk, even though the mechanism explaining this inverse correlation has not yet been clarified. The reduction in acid reflux that accompanies gastric atrophy has been proposed as a possible mechanism. A multicenter study showed that the four major risk factors - i.e. obesity, cigarette smoking, chronic GERD, and a diet low in fruits and vegetables - collectively were found in $79 \%$ (95\% CI: 66-87\%) of EA cases [20]. 


\section{The steps in Barrett's carcinogenesis}

Adenocarcinoma of the esophagus may develop through a series of morphological stages: from metaplasia to increasing grade of dysplasia and eventually to adenocarcinoma (see Fig. 1 for the metaplasia-dysplasia-adenocarcinoma sequence) [21,22].

At the molecular level, compared to colorectal carcinogenesis this multistep process has not yet been well-defined [23]. However, Barrett's carcinogenesis has a similar multistep process consisting of genetic and epigenetic mutations, which over many years can lead to increasing genomic instability and eventually to an autonomous clone of cells with invasive and metastatic features. In fact the time course of the progression to adenocarcinoma is extremely variable.

\subsection{Gastric metaplasia}

The interaction between acid reflux and proliferating adult progenitor cells of the squamous epithelium results in a genetic switch that causes a columnar transformation of the epithelium and the subsequent appearance of cardiac metaplasia in the distal esophagus [22]. Columnar metaplasia in the esophagus without intestinal specialized epithelium is absolutely specific for GERD. This marker could be considered the first step of Barrett's carcinogenesis, but whether it is a criterion for inclusion in standardized endoscopic surveillance programs is still a matter for debate $[24,25]$. The aberrant expression of keratin 7 is an early marker of the connection between GERD and columnar-lined esophagus. The Aurora-A over-expression may be a confirmation of this relationship [26-28].

\subsection{Intestinal metaplasia}

Intestinal metaplasia cannot be considered a marker for cancer risk since it can be found in most cases of BE, although not all [29]. If esophageal dysplasia and adenocarcinoma are believed to develop on a background of intestinal metaplasia, this belief leads to the misconception that without metaplasia cancer risk may be low [30]. The presence of a columnar metaplasia of the esophagus implies cancer risk regardless the intestinal phenotype. Moreover, even in the absence of detectable goblet cells, Barrett's mucosa still expresses markers and shows ultrastructural features consistent with intestinal differentiation [31,32].

\subsection{Dysplasia}

Dysplasia can be considered a clear marker of cancer risk in BE. It implies architectural and cytological changes commonly associated with carcinomas. For this reason it is presumed that carcinoma could evolve from dysplasia. Pathologists frequently do not agree on the identification of mild and moderate (low-grade) dysplasia. However, there is better agreement on severe (high-grade) dysplasia (HGD)
[33]. As a focal pattern in dysplasia can often be found, reliable detection needs several biopsies. This requirement implies that the endoscopic surveillance process may be complex [34]. We know that dysplasia can progress to highergrade forms or invasive adenocarcinoma, and that in some cases dysplastic changes may regress to non-dysplastic tissue. Moreover, high-grade dysplasia may persist for years before progressing to invasion. For this reason dysplasia appears to be a limited marker for risk of cancer. However, no better biomarker is yet available, and high-grade dysplasia will remain a mainstay of risk assessment in BE for some time until newer technologies allow better risk assessment. In this diagnostic process the pathologist has a predominant role. In fact, when three pathologists agree on a diagnosis of lowgrade dysplasia (LGD), an elevated risk of progression exists, perhaps because dysplasia on which any three pathologists can agree is close to being high-grade [35].

\section{Endoscopic surveillance for patients with Barrett's esophagus: a matter of debate}

Surveillance refers to periodic testing to detect disease or potential disease in a person at high risk for disease (Fig. 2). For patients with BE, the aim is to detect esophageal adenocarcinoma or high-grade dysplasia so that an early therapeutic intervention can result in a better outcome for those undergoing surveillance [36]. To date, patients with BE undergoing surveillance receive an endoscopy every 3-5 years for Barrett's metaplasia without dysplasia, every year for low-grade dysplasia, and every 3 months for high-grade dysplasia if no invasive treatment is offered [25,37].

Patients with BE usually undergo endoscopic surveillance at regular intervals, for instance every $2-3$ years if no additional abnormal findings are found. As a consequence a patient could undergo as many as ten or more surveillance endoscopies during their life time. As stated above, the incidence of EA is low and the surveillance endoscopies in BE patients usually don't detect cancer [38].

Even if all patients with BE experience an increased risk of developing esophageal adenocarcinoma, there are some patients for whom surveillance would be unacceptable or inappropriate.

To assess a real benefit from surveillance it is necessary to evaluate its impact on reduction in mortality, earlier cancer detection, and cost-effectiveness balance. The main aim of a BE surveillance program should be the reduction of the mortality from esophageal adenocarcinoma [39]. Mortality related to esophageal cancer in patients with $\mathrm{BE}$ is about $5 \%$. Since most BE patients die of causes other than esophageal adenocarcinoma, it may not be appropriate to offer routine surveillance for BE [40,41]. For example, in elderly patients with $\mathrm{BE}$ the surveillance is not really useful because of the higher the risk of death from other conditions. One might still derive support for a surveillance program if there were evidence that early detection was associated with improved 


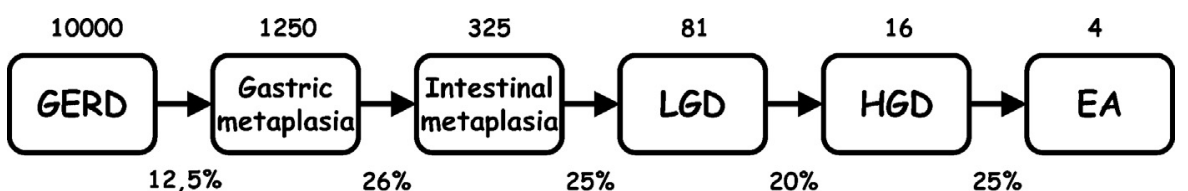

Fig. 1. Steps in Barrett's carcinogenesis. The expected number of cases for each step by histologically confirmed endoscopic evaluation are reported above the steps; the percentages progressing from one step to the next are inserted below. These data are extrapolated from the literature [22]. GERD, gastroesophageal reflux disease; LGD, low-grade dysplasia; HGD, high-grade dysplasia; EA, esophageal adenocarcinoma

survival. The 1-year and 5-year survival rates are higher in patients who have disease in situ than in those who have distant spread [42]. This suggests that a surveillance program has the potential to improve survival. Surveillance leads to detection of malignancy at an earlier stage and is a predictor of survival following surgery. Surveillance-detected adenocarcinomas were associated with lower-stage disease and improved survival compared with cancers that were not detected by surveillance $[43,44]$. BE surveillance may allow earlier detection of esophageal adenocarcinoma with consequent improvement in prognosis.

Furthermore, even if surveillance benefits some patients, the cost per patient may be such that it is not beneficial from a societal stand-point. As the incidence of esophageal adenocarcinoma is low, even in patients with $\mathrm{BE}$, there is a considerable cost associated with the identification of each case of adenocarcinoma. When a surveillance strategy is limited to older white males, smokers, and patients with more extensive BE or dysplasia, there is evidence that this leads to detection of disease when it is less advanced, with the subsequent potential improvement in prognosis.
To achieve the ideal treatment when high-grade dysplasia is found, a proper knowledge of the patient's characteristics is needed. Even if esophagectomy still remains the gold standard treatment for BE with high-grade dysplasia, minimally invasive endoscopic and ablative techniques have been recently adopted. The endoscopic procedures included in available options could be grouped into two major categories: endoscopic ablation of Barrett's mucosa that can be achieved by thermal, photodynamic and/or radiofrequency energy, and endoscopic mucosal resection. Randomized controlled trials are mandatory to confirm the effectiveness of these methods in preventing cancer development [45].

\section{Biomarkers to predict progression to esophageal adenocarcinoma}

Useful biomarkers in BE help to predict the onset of premalignant or malignant progression. Such markers are likely to distinguish a priori between people with low and high cancer risks. Ideal tests would be minimally invasive and

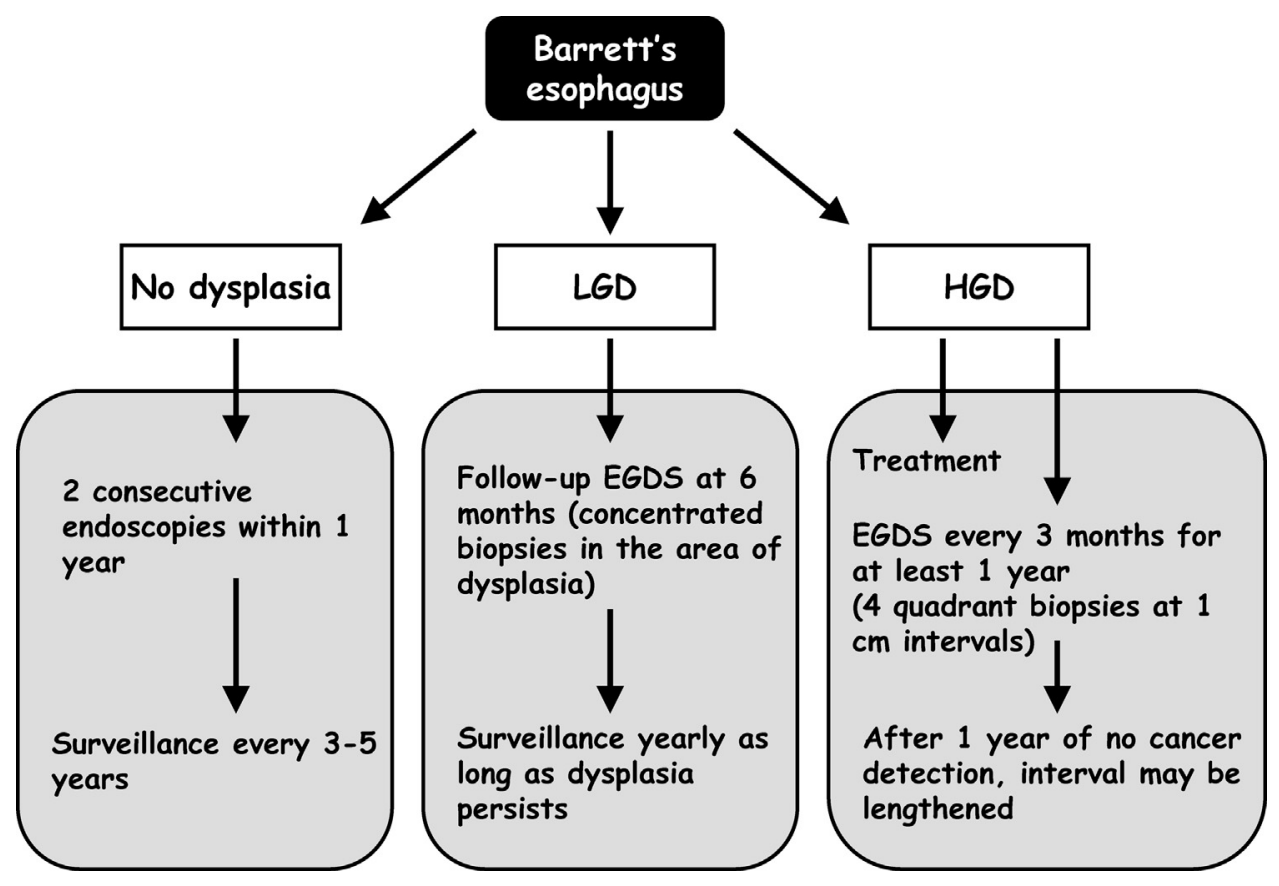

Fig. 2. Algorithm of endoscopic surveillance in subjects with Barrett's esophagus, as defined by the American Society for Gastrointestinal Endoscopy (ASGE) guidelines. LGD, low-grade dysplasia; HGD, high-grade dysplasia. 
cost-effective. It is desirable that the use of a biomarker in conjunction with other markers will allow improved sensitivity and specificity [46]. When a predictive biomarker is defined, it could be applied in selecting those patients with $\mathrm{BE}$ who could benefit from a more frequent endoscopic surveillance. The goal is to assess those predictive factors that may contribute to a better and quicker identification of patients with high-risk BE, so that surveillance strategies could be carried out.

Only 5\% of patients who present with esophageal adenocarcinoma already have a diagnosis of Barrett's metaplasia [47]. For this reason methods for the detection of early esophageal cancer need to be less invasive than endoscopic biopsies. However, to date the only way to stratify patients according to the risk of neoplasia of the esophagus involves histopathology, as until now only dysplasia has been strongly related with EA onset.

\subsection{DNA content abnormalities}

DNA content abnormalities occur in cancer development and are studied to improve our understanding of neoplastic transformation. Since normal cells contain 46 chromosomes $(2 \mathrm{~N})$, we refer to aneuploidy as the state in which cells have an abnormal number of chromosomes. Tetraploidy refers to cells that have double the number of chromosomes compared to normal cells $(4 \mathrm{~N})$.

In $\mathrm{BE}$, aneuploidy has been correlated with the progression to EA [48,49]. An increase in 4N (G2/tetraploid) cells predicts progression to aneuploidy. Moreover, the development of $4 \mathrm{~N}$ abnormalities is correlated with inactivation of the p53 gene.

Some authors have combined an approach using flow cytometry and histology with endoscopic biopsy. They demonstrated the role of aneuploidy and increased $4 \mathrm{~N}$-cell populations as biomarkers to identify those patients with $\mathrm{BE}$ at low and high risk of developing EA. More than $6 \%$ of cells with $4 \mathrm{~N}$ ploidy were considered abnormal. The relative risk of cancer for these patients compared to those below this cut-off value was 7.5 (95\% CI: 4-14). In addition, patients who had baseline aneuploidy had a relative risk of cancer of 5 (95\% CI: 2.7-9.4) compared to patients who did not have baseline aneuploidy.

\section{2. $p 53$}

p53 loss of heterozygosity ( $\mathrm{LOH}$ ) provides one of the most promising biomarkers to predict progression of BE. Silencing of p53 can occur via $\mathrm{LOH}$ or gene mutation. $\mathrm{LOH}$ of chromosome $17 \mathrm{p}$ (p53) significantly increased the risk of progression to cancer (relative risk of 16, 95\% CI: 6.2-39). Relative risk is higher when this biomarker is combined with aneuploidy ( $\mathrm{RR}=38.7,95 \% \mathrm{CI}: 10.8-138.5)$ [50,51]. The detection of LOH is complex and requires the collection of snap-frozen samples followed by extraction of DNA and an amplification step prior to polymerase chain reaction (PCR) analysis [52]. For this reason immunostaining for p53 was studied as an alternative method. The presence of p53 mutations can often cause protein accumulation, which allows for detection by immunohistochemistry. However, the efficacy of this as a biomarker is limited, because staining for $\mathrm{p} 53$ does not always correlate with mutations, e.g. when it results from deletion or truncation of $\mathrm{p} 53$ [53,54].

\subsection{Cell cycle markers}

Cyclin A and D have been also implicated in BE as biomarkers. The over-expression of Cyclin D (a proto-oncogene protein) in BE results in inappropriate phosphorylation and inactivation of p105-Rb. This phenomenon may be correlated with the predisposition to neoplastic transformation and cancer development. For this reason it was considered for studies on using it as a biomarker for the identification of those patients with $\mathrm{BE}$ at high risk of developing EA [55]. In a case-control study it was shown that histochemical positivity for cyclin D predicts higher probability of developing EA (OR: $6.85,95 \%$ CI: 1.57-29.91) [56]. However, these results were not confirmed in another larger population-based case-control study, where immunohistochemical detection of p53 was shown to be a useful biomarker for malignant progression in BE [53]. In a case-control study, surface expression of cyclin A in $\mathrm{BE}$ samples has been shown to be correlated with degree of dysplasia. Those patients whose biopsies express cyclin A had a higher probability of progressing to EA (OR: 7.5, 95\% CI: 1.8-30.7) [57]. Prospective studies are required to determine properly the usefulness of cyclins as predictive biomarkers.

\subsection{Epigenetic changes}

Epigenetic changes - including hypomethylation, hypermethylation, and alteration of histone complexes - seem to be implicated in Barrett's carcinogenesis. In particular, hypermethylation of the promoter $\mathrm{CpG}$ island of tumor suppressor genes such as CDKN2A (p16), APC, CDH1 (E-cadherin), and ESR1 (ER, estrogen receptor $\alpha$ ) induces transcriptional silencing. Hypermethylation of these genes is usually found in a large contiguous field, suggesting either a concerted methylation change associated with metaplasia or a clonal expansion of cells with abnormal hypermethylation $[58,59]$. In patients with non-dysplastic $\mathrm{BE}$ and low-grade dysplasia methylation of p16 (OR: 1.74, 95\% CI: 1.33-2.20), RUNX3 (OR: $1.80,95 \%$ CI: 1.08-2.81) and HPP1 (OR: 1.77, 95\% CI: 1.06-2.81) were observed as independent risk factors for progression to high-grade dysplasia and EA [60]. A methylation panel including eight genes could accurately determine the risk of progression in patients with $\mathrm{BE}$, as shown in a retrospective study. The promoter methylation levels of those eight genes were quantified by methylation-specific PCR in patients who did not progress compared to those 
who did progress to high-grade dysplasia or EA. ROC curves of the eight-gene methylation panel reached a specificity of $90 \%$ [61]. Another study combined four epigenetic (normalized methylation values for p16, HPP1, and RUNX3 and methylation index) and three clinical (patient's gender, BE segment length, pathological assessment) parameters to stratify the risk of progression to high-grade dysplasia and EA. Progression-free survivals differed significantly among the three risk groups (high, intermediate and low risk) [38]. The mechanisms by which a high methylation index contributes to carcinogenesis has not yet been clarified. Some hypotheses have been proposed to explain this phenomenon: (1) methylator phenotype-positive tumors are usually hypermethylated in the promoter regions of other genes, including tumor suppressor genes (such as APC, CDH1, TIMP3, and others); (2) a methylator phenotype induces inactivation of the hMLH1 gene by promoter hypermethylation, which in BE may causes microsatellite instability in the coding regions of the tumor suppressor genes; (3) a methylator phenotype may be associated with chromatin remodeling; and (4) methylated cytosines are hotspots for mutations, for example in the p53 gene.

These studies indicate that changes in DNA methylation occur early in Barrett's carcinogenesis. For this reason epigenetics could be useful as biomarkers to identify those patients who are likely to progress to dysplasia and EA. However, these techniques are far too technically demanding and timeconsuming for routine utilization in the clinic $[62,63]$.

\subsection{CDX genes}

CDX1 and CDX2 are transcription factors with a role in the development of intestinal phenotypes of gastrointestinal cells. In fact the gastric mucosa usually does not express these proteins; its epithelium becomes metaplastic through the genetic engineering of gastric cells to express their genes $[64,65]$. Cdx 1 and $\mathrm{Cdx} 2$ mediate the expression of cell adhesion proteins and subsequent maintenance of morphology and polarity in intestinal cells [66].

GERD-related damage to tight junctions between squamous cells is mediated by acid. As a consequence permeability increases with dilation of intercellular spaces. Undifferentiated cells in the basal layer of the epithelium are exposed to acid, bile salts and inflammatory mediators. By this mechanism these cells express CDX genes, and possibly also by epigenetic changes. Increased expression of these morphogenetic genes mediates the expression of homeotic genes that direct the squamous-to-columnar cell metaplasia characteristic of Barrett's esophagus. This hypothesis is support by the demonstration of high levels of CDX1 and CDX2 in the intestinal-type cells of Barrett's metaplasia [67,68]. For this reason a role for the measurement of CDX expression levels in esophageal squamous epithelium to predict development of BE in patients with GERD has been proposed [4].

\section{The microRNAs: a new way to understand carcinogenesis}

\subsection{Definition of microRNAs and their role in cancer}

MicroRNAs (miRNAs) are small (21-24 nucleotides long), endogenous, non-coding RNAs. Their direct effect is post-transcriptional gene silencing. Target mRNAs are recognized by base complementarity [69]. MiRNA genes are encoded in introns or exons of a protein-encoding gene or in the intergenic regions, and it has been estimated that they regulate up to $30 \%$ of human genes [70]. MiRNAs function as regulatory molecules in a wide variety of fundamental cellular processes, such as proliferation, death, differentiation, motility and invasiveness [71]. Aberrant expression of miRNAs has been observed in a diversity of pathological events. Importantly, deregulation or genetic changes of miRNAs have been critically implicated in the pathogenesis of most human cancers [72].

MiRNA biogenesis in the human cell is a multistep complex process that begins in the nucleus, where miRNA genes are transcribed by RNA polymerase II into long primary miRNAs (pri-miRNAs). Pri-miRNAs are subsequently cleaved into smaller, stem-looped, hairpin-like miRNA precursors (pre-miRNAs) of $\sim 70 \mathrm{nt}$ in length by an RNaseIII-type enzyme Drosha. Pre-miRNAs are exported from the nucleus into the cytoplasm by Exportin-5. In the cytoplasm, pre-miRNAs are then cleaved by Dicer ribonuclease. A single RNA strand is transferred to an argonaute protein within the RNA-induced silencing complex (RISC). The mature miRNA strand is preferentially incorporated into a microRNA-induced silencing complex (miRISC), while the other strand of miRNA is degraded by the RISC. The miRNA strand guides the RISC to its mRNA target containing a complementary sequence to the mature miRNA and subsequently induces the cleavage or silencing of the target mRNA [73].

Single miRNAs and specific miRNA expression profiles have been identified as tumor-related. MiRNAs can be categorized in two groups based on their functional relevance. MiRNAs with an oncogenic effect are defined as oncogenic miRNAs (oncomiRs). Those having a role as tumor suppressors are categorized as tumor-suppressive miRNAs (ts-miRs). In normal cells, ts-miRs are highly expressed and downregulate the expression of oncogenic proteins, whereas in tumor cells ts-miRs are silenced, leading to up-regulation of oncogenic proteins. Conversely, oncomiRs are up-regulated in tumor cells, down-regulating the expression of tumorsuppressive proteins.

\subsection{Role of microRNA expression changes in Barrett's carcinogenesis}

Since a differential expression of miRNAs was observed between normal esophageal epithelium and cancer cells, a role for miRNAs in the identification of patients at risk of esophageal adenocarcinoma development has been suggested 
[74]. Several publications have described miRNA expression in BE and esophageal adenocarcinoma. Some of these studies performed miRNA microarray and q-PCR analysis of miRNA expression in squamous esophageal epithelia, normal gastric epithelia, BE with intestinal metaplasia, and esophageal adenocarcinoma, with the aim of identifying new biomarkers for the different steps of Barrett's carcinogenesis $[75,76]$. The choice of including normal squamous esophageal epithelium in evaluating the differential miRNA expression profiles in the different steps of Barrett's carcinogenesis is questionable. In fact, the squamous phenotype of the esophageal epithelium has no correlation with EA, but it should only be compared with esophageal squamouscell cancer. To clarify this point studies are needed on the differentiation switch of progenitor cells from squamous to columnar-lined phenotypes and the correlation of this switch with carcinogenesis.

MiRNAs with differential expression patterns included miR-21, miR-143, miR-145, miR-194, miR-203, miR-205 and miR-215. However, various studies have also been performed to clarify the molecular consequences of the aberrant miRNA expression.

MiR-21 expression is up-regulated in BE and esophageal adenocarcinoma, compared with squamous esophageal epithelia, as found in other solid tumors [77]. Elevated miR21 has been implicated in many cellular processes required for neoplastic development and progression. Some authors have argued that the up-regulation of miR-21 in a metaplastic columnar epithelium may provide a selective advantage to cells for neoplastic development to esophageal adenocarcinoma [78].

miR-194 is also up-regulated in BE and esophageal adenocarcinoma. Expression of miR-194 is regulated by HNF-1a, a transcription factor induced in BE and esophageal adenocarcinoma. During intestinal epithelial cell differentiation, miR-194 expression has been shown to be induced [79]. Since its expression level was found to be increased even in metastatic pancreatic cell lines, we could hypothesize that elevated miR-194 levels could favor both intestinal metaplasia in BE and tumor metastatic phenotype [80].

miR-143, miR-145 and miR-215 are down-regulated in esophageal adenocarcinoma. Similar results have been found in colon, gastric and lung cancer [81-83]. Some studies have helped to explain the mechanisms by which miR-143, 145, and -215 induce carcinogenesis. For example, miR-143 targets the KRAS oncogene, so that colorectal cancer cell growth is inhibited by suppression of the translation of KRAS mRNA transcripts [84]. Up-regulated miR-143 also seems to have a role in FAS-mediated apoptosis [85]. These findings imply that loss of miR-143 expression in esophageal adenocarcinoma could result in lack of KRAS regulation, which favors neoplastic development.

Since both miR-143 and miR-145 expression are correlated with $\mathrm{p} 53$, their loss may alter the apoptotic responses in the progression of $\mathrm{BE}$ to esophageal adenocarcinoma $[86,87]$.
miR-215 together with miR-192 regulate cell cycle events through their ability to induce cell cycle arrest. For this reason the loss of miR-215 expression causes a reduction in the ability of cells to regulate proliferation, with the subsequent advantage to neoplastic clones [88]. On the basis of these findings we may define miR-143, -145 and -215 as tumor suppressors, with loss of expression contributing to the development of esophageal adenocarcinoma.

Two miRNAs recently showed roles as biomarkers in Barrett's carcinogenesis. miR-31 was found to be downregulated in both HGD and EA, probably as a consequence of the transition from BE to HGD. miR-375 showed marked down-regulation exclusively in EA and not in BE or HGD lesions. For this reason it could be considered a marker of progression to invasive carcinoma. miR-31 and miR-375 were proposed respectively as early and late biomarkers of malignant progression from Barrett's esophagus [89].

MiR-200 family members were also found to be downregulated in BE-derived high-grade dysplastic cell lines compared to a cell line derived from benign Barrett's epithelium. These miRNAs target ZEB1 and ZEB2, E-cadherin transcriptional repressors. ZEB1 and ZEB2 expression was significantly higher in esophageal adenocarcinoma compared to Barrett's esophagus epithelium from patients without cancer or dysplasia [90] (Table 1).

\subsection{Hypotheses for the causes of changes in microRNA expression}

The studies reported here show the possible mechanisms by which the miRNA variations in BE could modify the risk of developing esophageal adenocarcinoma. These hypotheses could be made from the findings of other studies which did not consider esophageal carcinogenesis [91]. However, to date very little is known about the possible mechanisms which induce miRNA variations in esophageal epithelium. For this reason some hypotheses could be proposed to induce further researches in this field. We provide some possible explanations, which need verification by specific studies: (1) polymorphisms could modify the basal levels of specific miRNAs which have a role in cancer susceptibility through advantage to neoplastic clones; (2) the genetic mutations of miRNA genes as a consequence of DNA damage could influence miRNA gene transcription; (3) the altered expression of some oncogenes and tumor suppressor genes in the various steps of the carcinogenetic process could modify the transcription of specific miRNA genes; or (4) microenvironmental factors could induce modifications in the pathway of miRNA biosynthesis.

If this last hypothesis can be demonstrated we could suppose that miRNAs could mediate in the connection between the action of carcinogenetic factors and the development of cancer clones bearing mutations in oncogenes or tumor suppressor genes. 
Table 1

miRNAs with differential expression in Barrett's esophagus (BE) and esophageal adenocarcinoma (EA). The known functions for each miRNA have been reported.

\begin{tabular}{|c|c|c|c|c|}
\hline & Ref. & $\mathrm{BE}$ & EA & Function \\
\hline $\operatorname{miR}-21$ & {$[77,78]$} & $\uparrow$ & $\uparrow$ & Implicated in many cellular processes required for neoplastic development and progression \\
\hline miR-194 & {$[79,80]$} & $\uparrow$ & $\uparrow$ & Implicated in intestinal epithelial cell differentiation \\
\hline $\operatorname{miR}-143$ & {$[84-87]$} & - & $\downarrow$ & $\begin{array}{l}\text { Suppression of translation of KRAS mRNA transcripts } \\
\text { A role in FAS-mediated apoptosis } \\
\text { Correlation with p53 }\end{array}$ \\
\hline miR-145 & {$[82,83]$} & - & $\downarrow$ & Correlation with p53 \\
\hline miR-215 & {$[81,88]$} & - & $\downarrow$ & Reduction in the ability of cells to regulate proliferation \\
\hline miR-31 & [89] & $\downarrow$ & $\downarrow$ & Early biomarkers of malignant progression from Barrett's esophagus \\
\hline miR-375 & [89] & - & $\downarrow$ & Late biomarkers of malignant progression from Barrett's esophagus \\
\hline miR-200 family & [90] & - & $\downarrow$ & Target ZEB1 and ZEB2, E-cadherin transcriptional repressors \\
\hline
\end{tabular}

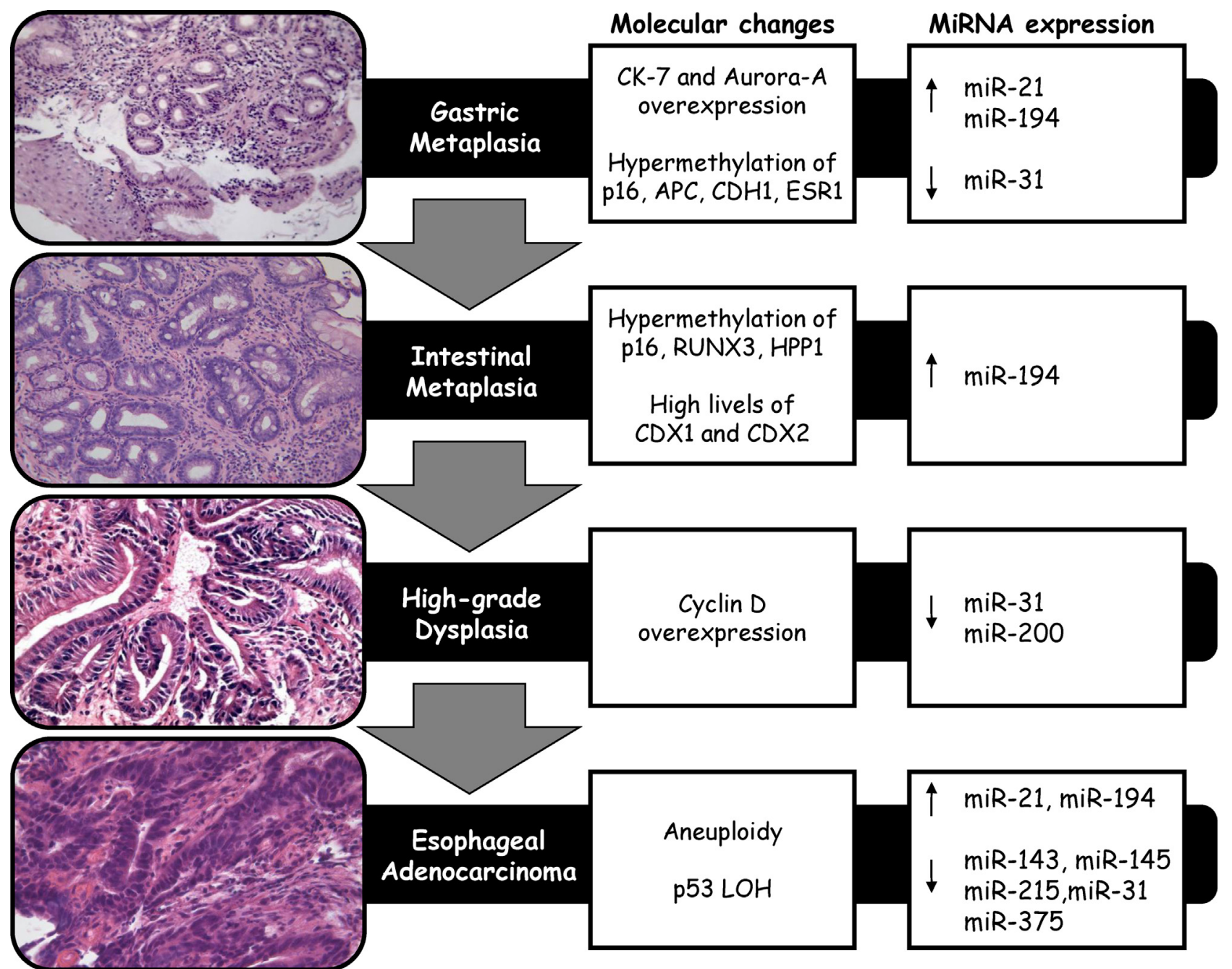

Fig. 3. The steps of progression toward esophageal adenocarcinoma. The correlation between molecular changes and miRNA expression are reported.

\section{Conclusions}

BE is a medical condition correlated with GERD, since acid reflux seems able to induce columnar metaplasia in the distal esophageal epithelium. Little is known about the cellular and molecular mechanisms which correlate with this phenotypic change. The incidence of EA appears to be on the increase, and this epidemiological phenomenon could be related to deteriorating lifestyle in Western countries. Because we know that dysplasia and adenocarcinoma arise in those patients with columnar metaplasia, BE has been considered as a predisposing condition for cancer. For 
this reason subjects with this condition may benefit from surveillance by endoscopy and consequent early diagnosis of esophageal cancer. The practical experience shows that longterm surveillance yields just a few cancer diagnoses despite the great economic cost and the discomfort for those patients who accept endoscopy. Some researchers are trying to find molecular alterations which could help in the selection of high-risk subjects as candidates for intensive surveillance. So far various alterations have been found which may allow this goal to be reached. These include DNA content abnormalities, p53 loss of heterozygosity, cell cycle markers, and epigenetic changes. Recently miRNAs have also been studied to identify significant differences between normal esophageal mucosa, metaplasia, dysplasia and adenocarcinoma (Fig. 3). However, the results of these studies are quite heterogeneous, and a specific pattern of miRNA expression related to high cancer risk has not yet been found. These findings provide an insight into potential molecular mechanisms to explain the development of adenocarcinoma from Barrett's metaplasia. miRNAs which show significant variations in dysplasia and adenocarcinoma should have a role in regulation of proliferation, and their alteration confers an advantage on neoplastic clonal expansion. To date it is not clear what leads to alteration in levels of miRNA expression. For this reason the question arises, analyzing the role of molecular changes in Barrett's carcinogenesis, including genetic, epigenetic and miRNA variations: which came first, the chicken or the egg? This conundrum refers to the possibility that those molecular alterations known to characterize the different steps of carcinogenesis could be consequences of microenvironmental exposure to carcinogenetic agents and predisposing factors for neoplastic transformation and clonal selection at the same time. We suppose that the solution of this question could clarify the reason why adenocarcinoma arises in BE.

\section{Conflicts of interest}

The authors have no conflicting interests to disclose.

\section{Reviewers}

Juan Iovanna, M.D. Ph.D., INSERM, Unité 624, Stress Cellulaire, Parc Scientifique et Technologique de Luminy, F-13288 Marseille Cedex 9, France.

Wainer Zoli, Ph.D., Department of Medical Oncology, Morgagni-Pierantoni Hospital, Forlì, Italy.

Jaffer A. Ajani, M.D., University of Texas MD Anderson Cancer Center, GI Medical Oncology Department, 1515 Holcombe Boulevard, Mailbox 426, Houston, TX 77030, United States.

Christian Diego Rolfo, M.D., Ph.D., MBHA, Senior Staff Member, University Hospital Antwerp, Oncology Department - Phase I-Early Clinical Trials Unit, Wilrijkstraat 1, B-2650 Edegem, Antwerpen, Belgium.

\section{References}

[1] Jenkins GJ, Doak SH, Parry JM, D’Souza FR, Griffiths AP, Baxter JN. Genetic pathways involved in the progression of Barrett's metaplasia to adenocarcinoma. The British Journal of Surgery 2002;89:824-37.

[2] Shaheen NJ, Richter JE. Barrett's oesophagus. Lancet 2009;373:850-61.

[3] Gillen P, Keeling P, Byrne PJ, Healy M, O’Moore RR, Hennessy TP. Implication of duodenogastric reflux in the pathogenesis of Barrett's oesophagus. The British Journal of Surgery 1988;75:540-3.

[4] Souza RF, Krishnan K, Spechler SJ. Acid, bile, and CDX: the ABCs of making Barrett's metaplasia. American Journal of Physiology 2008;295:G211-8.

[5] Playford RJ. New British Society of Gastroenterology (BSG) guidelines for the diagnosis and management of Barrett's oesophagus. Gut 2006;55:442.

[6] Ronkainen J, Aro P, Storskrubb T, et al. Prevalence of Barrett's esophagus in the general population: an endoscopic study. Gastroenterology 2005;129:1825-31.

[7] Dent J, El-Serag HB, Wallander MA, Johansson S. Epidemiology of gastro-oesophageal reflux disease: a systematic review. Gut 2005;54:710-7.

[8] Garud SS, Keilin S, Cai Q, Willingham FF. Diagnosis and management of Barrett's esophagus for the endoscopist. Therapeutic Advances in Gastroenterology 2010;3:227-38.

[9] Spechler SJ, Clinical practice. Barrett's esophagus. The New England Journal of Medicine 2002;346:836-42.

[10] Devesa SS, Blot WJ, Fraumeni Jr JF. Changing patterns in the incidence of esophageal and gastric carcinoma in the United States. Cancer 1998;83:2049-53.

[11] Parker SL, Tong T, Bolden S, Wingo PA. Cancer statistics, 1997. CA: A Cancer Journal for Clinicians 1997;47:5-27.

[12] Lambert R, Hainaut P. The multidisciplinary management of gastrointestinal cancer. Epidemiology of oesophagogastric cancer. Best Practice and Research 2007;21:921-45.

[13] Brown LM, Devesa SS, Chow WH. Incidence of adenocarcinoma of the esophagus among white Americans by sex, stage, and age. Journal of the National Cancer Institute 2008;100:1184-7.

[14] Coupland VH, Allum W, Blazeby JM, et al. Incidence and survival of oesophageal and gastric cancer in England between 1998 and 2007, a population-based study. BMC Cancer 2012;12:11.

[15] Yousef F, Cardwell C, Cantwell MM, Galway K, Johnston BT, Murray $\mathrm{L}$. The incidence of esophageal cancer and high-grade dysplasia in Barrett's esophagus: a systematic review and meta-analysis. American Journal of Epidemiology 2008;168:237-49.

[16] Hvid-Jensen F, Pedersen L, Drewes AM, Sorensen HT, Funch-Jensen P. Incidence of adenocarcinoma among patients with Barrett's esophagus. The New England Journal of Medicine 2011;365:1375-83.

[17] Kubo A, Corley DA. Body mass index and adenocarcinomas of the esophagus or gastric cardia: a systematic review and meta-analysis. Cancer Epidemiology, Biomarkers and Prevention 2006;15:872-8.

[18] Hampel H, Abraham NS, El-Serag HB. Meta-analysis: obesity and the risk for gastroesophageal reflux disease and its complications. Annals of Internal Medicine 2005;143:199-211.

[19] Whiteman DC, Sadeghi S, Pandeya N, et al. Combined effects of obesity, acid reflux and smoking on the risk of adenocarcinomas of the oesophagus. Gut 2008;57:173-80.

[20] Engel LS, Chow WH, Vaughan TL, et al. Population attributable risks of esophageal and gastric cancers. Journal of the National Cancer Institute 2003;95:1404-13.

[21] Jankowski JA, Wright NA, Meltzer SJ, et al. Molecular evolution of the metaplasia-dysplasia-adenocarcinoma sequence in the esophagus. The American Journal of Pathology 1999;154:965-73.

[22] Chandrasoma PT, Der R, Ma Y, Peters J, Demeester T. Histologic classification of patients based on mapping biopsies of the gastroesophageal junction. The American Journal of Surgical Pathology 2003;27:929-36. 
[23] Vogelstein B, Fearon ER, Hamilton SR, et al. Genetic alterations during colorectal-tumor development. The New England Journal of Medicine 1988;319:525-32.

[24] Guidelines for the diagnosis and management of Barrett's columnarlined oesophagus. British Society of Gastoenterology (BSG); 2005.

[25] Wang KK, Sampliner RE. Updated guidelines 2008 for the diagnosis, surveillance and therapy of Barrett's esophagus. The American Journal of Gastroenterology 2008;103:788-97.

[26] Agnese V, Cabibi D, Calcara D, et al. Aurora-A overexpression as an early marker of reflux-related columnar mucosa and Barrett's oesophagus. Annals of Oncology 2007;18(Suppl. 6):vi110-5.

[27] Cabibi D, Fiorentino E, Pantuso G, et al. Keratin 7 expression as an early marker of reflux-related columnar mucosa without intestinal metaplasia in the esophagus. Medical Science Monitor 2009;15:CR203-10.

[28] Cabibi D, Fiorentino E. Columnar-lined esophagus CK7 positive is an early marker of gastroesophageal reflux disease. The American Journal of Surgical Pathology 2011;35(5):773 [author reply 773-4].

[29] Kelty CJ, Gough MD, Van Wyk Q, Stephenson TJ, Ackroyd R. Barrett's oesophagus: intestinal metaplasia is not essential for cancer risk. Scandinavian Journal of Gastroenterology 2007;42:1271-4.

[30] Takubo K, Aida J, Naomoto Y, et al. Cardiac rather than intestinaltype background in endoscopic resection specimens of minute Barrett adenocarcinoma. Human Pathology 2009;40:65-74.

[31] Riddell RH, Odze RD. Definition of Barrett's esophagus: time for a rethink-is intestinal metaplasia dead? The American Journal of Gastroenterology 2009;104:2588-94.

[32] Dent J. Barrett's esophagus: a historical perspective, an update on core practicalities and predictions on future evolutions of management. Journal of Gastroenterology and Hepatology 2011;26(Suppl. 1):11-30.

[33] Spechler SJ. Dysplasia in Barrett's esophagus: limitations of current management strategies. The American Journal of Gastroenterology 2005;100:927-35.

[34] Abela JE, Going JJ, Mackenzie JF, McKernan M, O’Mahoney S, Stuart RC. Systematic four-quadrant biopsy detects Barrett's dysplasia in more patients than nonsystematic biopsy. The American Journal of Gastroenterology 2008;103:850-5.

[35] Skacel M, Petras RE, Gramlich TL, Sigel JE, Richter JE, Goldblum JR. The diagnosis of low-grade dysplasia in Barrett's esophagus and its implications for disease progression. The American Journal of Gastroenterology 2000;95:3383-7.

[36] Dulai GS. Surveying the case for surveillance. Gastroenterology 2002;122:820-3.

[37] Spechler SJ, Sharma P, Souza RF, Inadomi JM, Shaheen NJ. American Gastroenterological Association technical review on the management of Barrett's esophagus. Gastroenterology 2011;140:e18-52, quiz e13.

[38] Sato F, Jin Z, Schulmann K, et al. Three-tiered risk stratification model to predict progression in Barrett's esophagus using epigenetic and clinical features. PloS ONE 2008;3:e1890.

[39] Macdonald CE, Wicks AC, Playford RJ. Final results from 10 year cohort of patients undergoing surveillance for Barrett's oesophagus: observational study. British Medical Journal 2000;321:1252-5 [Clinical Research Ed.].

[40] Conio M, Blanchi S, Lapertosa G, et al. Long-term endoscopic surveillance of patients with Barrett's esophagus. Incidence of dysplasia and adenocarcinoma: a prospective study. The American Journal of Gastroenterology 2003;98:1931-9.

[41] Thomas T, de Caestecker JS. Surveillance in Barrett's oesophagus. European Journal of Gastroenterology and Hepatology 2006;18: 585-8.

[42] Eloubeidi MA, Mason AC, Desmond RA, El-Serag HB. Temporal trends (1973-1997) in survival of patients with esophageal adenocarcinoma in the United States: a glimmer of hope? The American Journal of Gastroenterology 2003;98:1627-33.

[43] Fountoulakis A, Zafirellis KD, Dolan K, Dexter SP, Martin IG, SueLing HM. Effect of surveillance of Barrett's oesophagus on the clinical outcome of oesophageal cancer. The British Journal of Surgery 2004;91:997-1003.

[44] Corley DA, Levin TR, Habel LA, Weiss NS, Buffler PA. Surveillance and survival in Barrett's adenocarcinomas: a population-based study. Gastroenterology 2002;122:633-40.

[45] Lekakos L, Karidis NP, Dimitroulis D, Tsigris C, Kouraklis G, Nikiteas N. Barrett's esophagus with high-grade dysplasia: focus on current treatment options. World Journal of Gastroenterology 2011:17:4174-83.

[46] Preston SL, Jankowski JA. Drinking from the fountain of promise: biomarkers in the surveillance of Barrett's oesophagus - the glass is half full! Gut 2006;55:1377-9.

[47] Dulai GS, Guha S, Kahn KL, Gornbein J, Weinstein WM. Preoperative prevalence of Barrett's esophagus in esophageal adenocarcinoma: a systematic review. Gastroenterology 2002;122:26-33.

[48] Reid BJ, Levine DS, Longton G, Blount PL, Rabinovitch PS. Predictors of progression to cancer in Barrett's esophagus: baseline histology and flow cytometry identify low- and high-risk patient subsets. The American Journal of Gastroenterology 2000;95:1669-76.

[49] Galipeau PC, Cowan DS, Sanchez CA, et al. 17p (p53) allelic losses, 4N (G2/tetraploid) populations, and progression to aneuploidy in Barrett's esophagus. Proceedings of the National Academy of Sciences of the United States of America 1996;93:7081-4.

[50] Reid BJ, Prevo LJ, Galipeau PC, et al. Predictors of progression in Barrett's esophagus II: baseline 17p (p53) loss of heterozygosity identifies a patient subset at increased risk for neoplastic progression. The American Journal of Gastroenterology 2001;96:2839-48.

[51] Galipeau PC, Li X, Blount PL, et al. NSAIDs modulate CDKN2A, TP53, and DNA content risk for progression to esophageal adenocarcinoma. PLoS Medicine 2007;4:e67.

[52] Paulson TG, Galipeau PC, Reid BJ. Loss of heterozygosity analysis using whole genome amplification, cell sorting, and fluorescence-based PCR. Genome Research 1999;9:482-91.

[53] Murray L, Sedo A, Scott M, et al. TP53 and progression from Barrett's metaplasia to oesophageal adenocarcinoma in a UK population cohort. Gut 2006;55:1390-7.

[54] Weston AP, Banerjee SK, Sharma P, Tran TM, Richards R, Cherian R. p53 protein overexpression in low grade dysplasia (LGD) in Barrett's esophagus: immunohistochemical marker predictive of progression. The American Journal of Gastroenterology 2001;96: $1355-62$.

[55] Trudgill NJ, Suvarna SK, Royds JA, Riley SA. Cell cycle regulation in patients with intestinal metaplasia at the gastro-oesophageal junction. Molecular Pathology 2003;56:313-7.

[56] Bani-Hani K, Martin IG, Hardie LJ, et al. Prospective study of cyclin D1 overexpression in Barrett's esophagus: association with increased risk of adenocarcinoma. Journal of the National Cancer Institute 2000;92:1316-21.

[57] Lao-Sirieix P, Lovat L, Fitzgerald RC. Cyclin A immunocytology as a risk stratification tool for Barrett's esophagus surveillance. Clinical Cancer Research 2007;13:659-65.

[58] Eads CA, Lord RV, Kurumboor SK, et al. Fields of aberrant CpG island hypermethylation in Barrett's esophagus and associated adenocarcinoma. Cancer Research 2000;60:5021-6.

[59] Wang JS, Guo M, Montgomery EA, et al. DNA promoter hypermethylation of 16 and APC predicts neoplastic progression in Barrett's esophagus. The American Journal of Gastroenterology 2009;104:2153-60.

[60] Schulmann K, Sterian A, Berki A, et al. Inactivation of p16, RUNX3, and HPP1 occurs early in Barrett's-associated neoplastic progression and predicts progression risk. Oncogene 2005;24:4138-48.

[61] Jin Z, Cheng Y, Gu W, et al. A multicenter, double-blinded validation study of methylation biomarkers for progression prediction in Barrett's esophagus. Cancer Research 2009;69:4112-5. 
[62] Eads CA, Lord RV, Wickramasinghe K, et al. Epigenetic patterns in the progression of esophageal adenocarcinoma. Cancer Research 2001;61:3410-8.

[63] Clark SJ, Harrison J, Paul CL, Frommer M. High sensitivity mapping of methylated cytosines. Nucleic Acids Research 1994;22:2990-7.

[64] Mutoh H, Satoh K, Kita H, et al. Cdx2 specifies the differentiation of morphological as well as functional absorptive enterocytes of the small intestine. The International Journal of Developmental Biology 2005;49:867-71.

[65] Silberg DG, Furth EE, Taylor JK, Schuck T, Chiou T, Traber PG. CDX1 protein expression in normal, metaplastic, and neoplastic human alimentary tract epithelium. Gastroenterology 1997;113: 478-86.

[66] Guo RJ, Suh ER, Lynch JP. The role of Cdx proteins in intestinal development and cancer. Cancer Biology and Therapy 2004;3: 593-601.

[67] Colleypriest BJ, Palmer RM, Ward SG, Tosh D. Cdx genes, inflammation and the pathogenesis of Barrett's metaplasia. Trends in Molecular Medicine 2009;15:313-22.

[68] Stairs DB, Kong J, Lynch JP. Cdx genes, inflammation, and the pathogenesis of intestinal metaplasia. Progress in Molecular Biology and Translational Science 2010;96:231-70.

[69] Garzon R, Calin GA, Croce CM. MicroRNAs in cancer. Annual Review of Medicine 2009;60:167-79.

[70] Bartel DP. MicroRNAs: genomics, biogenesis, mechanism, and function. Cell 2004;116:281-97.

[71] Carleton M, Cleary MA, Linsley PS. MicroRNAs and cell cycle regulation. Cell Cycle (Georgetown, TX) 2007;6:2127-32.

[72] Croce CM. Causes and consequences of microRNA dysregulation in cancer. Nature Reviews 2009;10:704-14.

[73] Lowery AJ, Miller N, McNeill RE, Kerin MJ. MicroRNAs as prognostic indicators and therapeutic targets: potential effect on breast cancer management. Clinical Cancer Research 2008;14:360-5.

[74] Feber A, Xi L, Luketich JD, et al. MicroRNA expression profiles of esophageal cancer. The Journal of Thoracic and Cardiovascular Surgery 2008;135:255-60 [discussion 60].

[75] Watson DI, Michael WB, Mayne MZGCDJH. MicroRNA expression profiles in Barrett's oesophagus. ANZ Journal of Surgery 2007;77:A45.

[76] Yang H, Gu J, Wang KK, et al. MicroRNA expression signatures in Barrett's esophagus and esophageal adenocarcinoma. Clinical Cancer Research 2009;15:5744-52.

[77] Krichevsky AM, Gabriely G. miR-21: a small multi-faceted RNA. Journal of Cellular and Molecular Medicine 2009;13:39-53.

[78] Smith CM, Watson DI, Michael MZ, Hussey DJ. MicroRNAs, development of Barrett's esophagus, and progression to esophageal adenocarcinoma. World Journal of Gastroenterology 2010;16:531-7.

[79] Hino K, Tsuchiya K, Fukao T, et al. Inducible expression of microRNA194 is regulated by HNF-1alpha during intestinal epithelial cell differentiation. RNA (New York, NY) 2008;14:1433-42.

[80] Mees ST, Mardin WA, Wendel C, et al. EP300 - a miRNA-regulated metastasis suppressor gene in ductal adenocarcinomas of the pancreas. International Journal of Cancer 2010;126:114-24.

[81] Braun CJ, Zhang X, Savelyeva I, et al. p53-Responsive micrornas 192 and 215 are capable of inducing cell cycle arrest. Cancer Research 2008;68:10094-104.

[82] Takagi T, Iio A, Nakagawa Y, Naoe T, Tanigawa N, Akao Y. Decreased expression of microRNA-143 and -145 in human gastric cancers. Oncology 2009;77:12-21.

[83] Liu X, Sempere LF, Galimberti F, et al. Uncovering growthsuppressive MicroRNAs in lung cancer. Clinical Cancer Research 2009;15:1177-83.

[84] Chen X, Guo X, Zhang H, et al. Role of miR-143 targeting KRAS in colorectal tumorigenesis. Oncogene 2009;28:1385-92.

[85] Akao Y, Nakagawa Y, Iio A, Naoe T. Role of microRNA-143 in Fasmediated apoptosis in human T-cell leukemia Jurkat cells. Leukemia Research 2009;33:1530-8.
[86] Spizzo R, Nicoloso MS, Lupini L, et al. miR-145 participates with TP53 in a death-promoting regulatory loop and targets estrogen receptoralpha in human breast cancer cells. Cell Death and Differentiation 2010;17:246-54.

[87] Suzuki HI, Yamagata K, Sugimoto K, Iwamoto T, Kato S, Miyazono K. Modulation of microRNA processing by p53. Nature 2009;460:529-33.

[88] Georges SA, Biery MC, Kim SY, et al. Coordinated regulation of cell cycle transcripts by p53-inducible microRNAs, miR-192 and miR-215. Cancer Research 2008;68:10105-12.

[89] Leidner RS, Ravi L, Leahy P, et al. The microRNAs, MiR-31 and MiR-375, as candidate markers in Barrett's esophageal carcinogenesis. Genes, Chromosomes and Cancer 2012;51:473-9.

[90] Smith CM, Watson DI, Leong MP, et al. miR-200 family expression is downregulated upon neoplastic progression of Barrett's esophagus. World Journal of Gastroenterology 2011;17:1036-44.

[91] Kwak PB, Iwasaki S, Tomari Y. The microRNA pathway and cancer. Cancer Science 2010;101:2309-15.

\section{Biographies}

Antonio Russo, M.D., is Associate Professor of Medical Oncology at University Medical School of Palermo, Department of Surgical and Oncological Sciences (Italy). From 2004 to July 2011 he has been an Adjunct Associate Professor and since August 2011 Adjunct Full Professor at Temple University's College of Science and Technology, Philadelphia (USA) and. Since 2001 he has been a coordinator with Prof D. Kerr (University of Oxford, UK) and Prof B. Iacopetta (Western Australia University) of the "CRCP53 International Collaborative Study". Since 2003 he has been an expert member of INSERM (Institut National de la Santè et de la Recherche Mèdical, France), since 2007 of Scientific Committee INCA (Institut National du Cancer, France) and of NWCRF (North West Cancer Research Fund, UK). He is member of Editorial board of Journal of Carcinogenesis \& Mutagenesis (since 2011) and World Journal of Gastrointestinal Oncology and World Journal of Clinical Oncology (since 2012). Since 2013 is Associate Editor of Journal of Solid Tumors. Since 2008 he has been Guest Editor of Annals of Oncology $(2006,2007)$. The central theme of his studies is translational research, meaning the application of molecular genetics in cancer management. He is PI in several national and international clinical trials. He is the author of more than 200 peer-reviewed publications listed on Medline-PubMed.

Giuseppe Bronte received his M.D. degree from University Medical School of Palermo (Italy) in 2004. His post-graduate specialty was in Medical Oncology in 2008. He received his Ph.D. degree in Experimental and Clinical Oncology from the same University in 2012. Co-investigator and Data manager in Multicenter Clinical Trials, managed by different Clinical Research Cooperative Groups, according to Good Clinical Practice (ITMO, SICOG, GOIM). He is a member of scientific societies and he is actively involved in the teaching and research of oncology fellows and students. He is author more than 20 publications in top-rated international cancer journals. 
Daniela Cabibi received a degree in Biology in 1984 and in Medicine in 1987 at the University of Palermo. Her postgraduate specialty was in Pathology and she also followed the course in breast pathology at the Department of Anatomic and Surgical Sciences, Faculty of Medicine of Palermo. In 1991 she performed a training at the University of Louvain, in Belgium. Since 2001 she worked as a researcher at the University of Palermo, Department of Pathology and since 2010 she is Associate Professor of Pathology at the Faculty of Medicine of the University of Palermo. She is author of more than 80 articles published on the most important international journal.

Viviana Bazan, Ph.D., is Aggregate Professor of General Pathology at University Medical School of Palermo, Department of Surgical and Oncological Sciences (Italy). From July 2008 to July 2011, she has been an Adjunct Assistant Professor and since August 2011 is Adjunct Associate Professor at Temple University's College of Science and Technology, Philadelphia (USA). She has been Co-Editor of Annals of Oncology (Volume 17, 2006 and Volume 18, 2007). Over the last few years, she has been implicated in clinical oncology research aimed at identifying biomolecular prognostic features and treatment response. In this context she has been concerned with the molecular genetics of sporadic, hereditary and familial tumors. She is the author of more than 120 publications in top-rated cancer journals.

Giuseppe Cicero, M.D. received his doctorate in clinical and experimental oncology from the University of Palermo School of Medicine. He is currently adjunct professor in medical oncology at the university of Palermo, and his scientific work focuses on molecular biology of cancer, immunology and target therapies. He is PI and Sub-I in several national and international clinical trials.

Alessandro Bertani, M.D., Ph.D. earned his medical degree at the University of Pavia, Italy in 1996. He performed his surgical residency in Bergamo, Italy at the Ospedali Riuniti Transplant Center. Between 2002 and 2004 he enrolled into additional training at the University of Pittsburgh, USA, serving a fellowship in Cardiothoracic Transplant Surgery and a fellowship in Minimally Invasive Thoracic Surgery. $\mathrm{He}$ also gained a Ph.D. degree in Microsurgery and Experimental Surgery at the University of Pavia. He is currently an Assistant Professor of Surgery at the University of Pittsburgh and chief of the Department of Thoracic Surgery and Lung Transplantation at ISMETT-UPMC Italy in Palermo, Italy. Here, he is constantly involved in clinical and experimental lung transplantation, pulmonary mediastinal and esophageal surgery. He has a specific interest in minimally invasive thoracic surgery. He is author and co-author of book chapters and peer-reviewed publications, mostly focusing on clinical and experimental topics of lung transplantation.

Sergio Rizzo received his M.D. degree from University Medical School of Palermo (Italy) in 2003. His post-graduate specialty was in Medical Oncology. He received his $\mathrm{PhD}$ degree in Oncopathology from the same University in 2011. He has been awarded a AIOM Foundation Fellowship and has just spent six months at Lee Moffitt Cancer Center in Tampa, FL (USA). He is author of more than 20 publications in top-rated international cancer journals.

Eugenio Fiorentino M.D. is Full Professor of Surgery at University School of Medicine of Palermo, Department of Surgical and Oncological Sciences. He is leading expert in gastroesophageal reflux disease and clinical director of the Esophageal Diseases Clinical Program at University Hospital Policlinico in Palermo. He has authored over 100 scientific publications mainly on gastroesophageal reflux disease and edited one book on acid reflux. Current area of research interest include gastroesophageal reflux, Barrett's esophagus, and esophageal cancer. 